\title{
Contrast-dependent interaction between steadily fixated lines
}

\author{
M. T. SWANSTON \\ Dundee College of Technology, Bell Street, Dundee DDI IHG, Scotland
}

\begin{abstract}
Disappearances of a steadily fixated line were shown to be reduced when it was paired with a like-oriented second line of the same luminance. The effect did not decline with $2.4 \mathrm{deg}$ of separation between the lines. No such stabilization was obtained if the second line was either brighter than the reference line or decremental with respect to the background. It was found that this selective interaction was not the result of either a reduction in the apparent brightness of the dim line by simultaneous contrast with the brighter one or of the different response requirements when viewing two dim lines as opposed to one. Disappearances in steady fixation can therefore indicate the existence of processes that are not apparent in other forms of steady visual stimulation.
\end{abstract}

Interactions between adjacent visual stimuli have been found for a wide range of perceptual characteristics, such as orientation (Carpenter \& Blakemore, 1973), simultaneous contrast (Ratliff, 1965), afterimage visibility (Atkinson, 1973), and binocular rivalry (Wade, 1973, 1976; Whittle, Bloor, \& Pocock, 1968). Interest in these effects has been increased by the fact that they may reflect the presence of reciprocal inhibition between elements in the visual system that are selectively responsive to particular perceptual features (e.g., Walker, 1978). Conclusions so drawn may therefore be comparable to those derived from studies of various spatial aftereffects (Weisstein, 1969). The present experiments investigated the manner in which the visibility of a steadily fixated line was modified by the presence, separation, and luminance of a second line simultaneously present in the visual field. Under suitable conditions, steady fixation of a visual stimulus results in periodic disappearances, which resemble both quantitatively and qualitatively the visibility cycles of afterimages and optically stabilized images (MacKinnon, Forde, \& Piggins, 1969).

Atkinson (1972) reported that the visibility of a linear afterimage was increased by the presence of a second line in the same orientation but was decreased if the lines were in different orientations. Both effects were found to decline up to separations of $2 \mathrm{deg}$. Similarly, Wade (1976) found that the visibility of the afterimage of a circular grating was increased by the concurrent presentation of a surround grating of the same orientation if both were seen by the same eye. Interactions between lines of similar orientation have also been reported by Schmidt, Cosgrove, and Brown (1972) for optically stabilized lines viewed after preadaptation to gratings in the same or in different orientations. Prior viewing of a like-oriented grating increased the frequency of fading of the stabilized line, which the authors suggested could have been due to selective adaptation of orientation-specific pattern elements in the visual system. Comparable data for steady fixation are limited (e.g., Roessel, 1966) but would provide information about the equivalence of the various methods of achieving steady visual stimulation. Insofar as steady fixation can be shown to reveal processes similar to those established for afterimages and optical stabilization, the method offers advantages over other procedures. In particular, with steady fixation, it is possible to manipulate the luminance of stimuli both within and between trials and thus to examine the dependence of stimulus visibility on characteristics whose control is impracticable with afterimages and confined to very few subjects with optical stabilization (e.g., Brown, Schmidt, Fulgham, \& Cosgrove, 1973).

\section{EXPERIMENT 1}

This experiment investigated the visibility of a reference line that was either viewed alone or paired with a second line at separations of .8 or $2.4 \mathrm{deg}$. The luminance of the second line was either the same as that of the reference line $\left(.86 \mathrm{~cd} / \mathrm{m}^{2}\right)$ or markedly brighter $\left(2.72 \mathrm{~cd} / \mathrm{m}^{2}\right)$. The latter value was such that it minimized the probability of disappearances with even the steadiest fixation. The five experimental conditions (see Figure 1 for summary) were expected to indicate any effect on the visibility of the reference line due to the presence, separation, and luminance of a second line.

\footnotetext{
Method

Apparatus. Subjects fixated a small black fixation point, located at the center of a back-projection screen that subtended $12 \times$ $16 \mathrm{deg}$ and whose luminance was set at $6.84 \mathrm{~cd} / \mathrm{m}^{2}$. Stimuli were presented as lines $(.8 \times .08 \mathrm{deg})$, superimposed as increments on
} 
A

.

$\mathbf{B}, \mathrm{C}$

D,E

Figure 1. Diagrammatic representation of the stimulus configurations employed in Experiment 1. In Conditions $C$ and $E$, the right-hand line was of higher luminance than the other (reference) line. The black dot represents the fixation point.

the screen in the same depth plane, by means of partially reflecting mirrors. An adjustable chinrest constrained the subject's head position, and two microswitch keys were provided for use with a subject's preferred hand. The frequency and duration of all combinations of key depressions were monitored by appropriate counters and timers.

Subjects. Twenty subjects took part in the experiment. All had normal or corrected vision, and none had a history of childhood strabismus (Forde \& MacKinnon, 1975). The sighting dominant eye was used for observations, the other being occluded.

Procedure. Each subject viewed all five stimulus configurations in a random order. Each trial lasted $120 \mathrm{sec}$, during which subjects were instructed to fixate the central dot as carefully as possible. Disappearances were reported by depressing, as appropriate, the two response keys provided for as long as a given line was completely invisible. Partial disappearances of a line were not therefore reported. A recovery interval of $120 \mathrm{sec}$ was given between each trial, so that a complete session was approximately $25 \mathrm{~min}$ long.

\section{Results and Discussion}

Table 1 summarizes the results for the reference line, in terms of the total time for which it was reported to disappear during a trial (disappearance time), and the mean duration of each period of disappearance (mean period).

When viewed alone (Condition $\mathrm{A}$ ), the reference line disappeared, in all, for $23.6 \mathrm{sec}$ and for about $4.5 \mathrm{sec}$ each time a disappearance took place. The most marked deviations from this pattern were found with Conditions $B$ and $D$, in which the second line was of the same luminance as the reference line, al- though at varying separations. The effects of the luminance and separation of the second line were examined by an analysis of variance, using subjects' difference scores between Condition $\mathbf{A}$ and each of the other conditions. For both disappearance time and mean period, there were significant effects of luminance $[\operatorname{Fs}(1,19)=9.8$ and 10.1 , respectively; both ps $<.01]$, but none due to separation $[\mathrm{Fs}(1,19)=.05$ and .02], and there was no significant interaction $[F s(1,19)=1.54$ and .27$]$. The reference line therefore showed a consistently increased visibility when paired with a second line of the same luminance, irrespective of separation up to $2.4 \mathrm{deg}$. When the second line was of a higher luminance, no such stabilizing effect occurred, again regardless of separation.

Conditions B and D also provided a measure of the effect of separation on the joint disappearances of the components of a two-line pattern, which Atkinson (1973) found to decline by around $25 \%$ as the separation of two like-oriented lines was increased from 1 to $4 \mathrm{deg}$. Although a number of measures of such synchronized visibility have been derived (e.g., Atkinson, 1973; Mackinnon et al., 1969), the ratio of whole pattern disappearance time to the time for which the pattern shows any type of fragmentation, which can be termed "coherence"' (Swanston, 1979), provides an adequate index of this characteristic. Using this measure, there was a significant difference between the two conditions. For Condition B, coherence was .49 , and for Condition $D$, it was .18 $[\mathrm{t}(19)=3.66, \mathrm{p}<.01]$. With a more peripheral location of the second line, disappearances of one line or the other increased, but the proportion of these that were of both lines together showed a marked decline. This was broadly equivalent to the reported findings for similarity patterned afterimages (Atkinson, 1973; Owen \& Chalfant, 1970) and optically stabilized images (Pritchard, Heron, \& Hebb, 1960) and thus provided support for the equivalence of various methods of steady stimulation.

\section{EXPERIMENT 2}

In Experiment 1, the reference line showed a reduced disappearance time when paired with another

Table 1

Response Measures for the Stability of the Reference Line in Experiment 1

\begin{tabular}{lrrrrr}
\hline & \multicolumn{5}{c}{ Stimulus Condition } \\
\cline { 2 - 6 } & $\mathrm{A}$ & \multicolumn{1}{c}{ B } & \multicolumn{1}{c}{ C } & \multicolumn{1}{c}{ D } & \multicolumn{1}{c}{ E } \\
\hline Disappearance Times (S) & 23.6 & 15.9 & 21.5 & 13.8 & 26.7 \\
Mean Period (s) & 4.5 & 3.1 & 4.1 & 2.8 & 5.4 \\
\hline
\end{tabular}

Note-Stimulus conditions: $A$, single line; $B, .8$-deg separation, same luminance; $C, .8$-deg separation, second line higher luminance; $D$, 2.4-deg separation, same luminance; $E$, 2.4-deg separation, second line higher luminance. 
line of the same luminance, as opposed to being viewed on its own or with a second line of higher luminance. A possible explanation of this effect was that the bright second line might have reduced the apparent brightness of the reference line by means of simultaneous contrast and thus led to a higher probability of disappearance. This account would predict a higher disappearance time for the reference line paired with a bright line than when the reference line alone was viewed and also a declining effect with increasing separation (Bishop, Coombs, \& Henry, 1971; Leibowitz, Mote, \& Thurlow, 1953), neither of which was the case. However, the explanation required a direct test, and this was carried out in Experiment 2 by presenting the reference line with a second line that could be of the same luminance (Condition A), of intermediate luminance (Condition B), of the same higher luminance as that used in Experiment 1 (Condition $\mathrm{C}$ ), or of decremental contrast with respect to the background (Condition D). Comparisons were planned between Condition $\mathbf{A}$ as a control and each of the other conditions.

\section{Method}

Apparatus. Stimuli were presented with the same apparatus as in Experiment 1, with the addition of a sheet of clear glass in front of the background screen. For Condition D, this was replaced by a sheet of glass carrying a single opaque black line of the same dimensions as the luminous lines. In Condition $B$, the luminance of the second line was set by neutral density filters to $2.2 \mathrm{~cd} / \mathrm{m}^{2}$.

Subjects. Fifteen subjects took part in the experiment. All had normal or corrected vision and no history of strabismus. The sighting dominant eye was used for observations.

Procedure. Subjects viewed the four stimulus configurations in a balanced order. Response instructions and the conduct of trials were the same as in Experiment 1.

\section{Results and Discussion}

Table 2 summarizes the results for the reference line in the same terms as Table 1 . The overall pattern of results was similar to that obtained with Experiment 1 , in that the reference line was more stable when paired with another line of the same luminance than when paired with either a brighter or a darker second line. Evaluation of the results by Dunnett's procedure (Kirk, 1968) showed that the critical difference between Condition $\mathbf{A}$ and any other condition

Table 2

Response Measures for the Stability of the Reference Line in Experiment 2

\begin{tabular}{lrrrr} 
& \multicolumn{4}{c}{ Stimulus Condition } \\
\cline { 2 - 5 } & A & \multicolumn{1}{c}{ B } & \multicolumn{1}{c}{ C } & \multicolumn{1}{c}{ D } \\
\hline Disappearance Times (S) & 9.4 & 13.3 & 17.9 & 15.5 \\
Mean Period (s) & 2.1 & 2.8 & 3.3 & 3.7 \\
\hline
\end{tabular}

Note-Stimulus conditions: $A$, single line; $B$, second line intermediate luminance; $C$, second line higher luminance; $D$, second line decrement. needed for significance $(\mathrm{df}=4,42, \mathrm{p}<.05)$ was 5.4 for disappearance time and 1.1 for mean period. This was exceeded on both measures in Conditions C and D but not in Condition $B$, which, although differing in the same direction, did not reach significance. Condition $\mathrm{C}$ therefore replicated the result of Experiment 1 , and Condition $\mathrm{D}$ demonstrated that this absence of an interaction also occurred when the second line was of decremental contrast. Since the latter would be expected to have, if anything, opposite effects on the perceived contrast of the reference line, an explanation in terms of simultaneous brightness contrast was therefore eliminated. The interaction between the two lines was thus found to be dependent on relative rather than absolute luminance, but the origins of the effect could not be further identified.

\section{EXPERIMENT 3}

When a dim line is paired with a bright line, the latter is unlikely to undergo subjective disappearances, and a subject has effectively to report only the changes in visibility of the dim line. This task therefore differs from that required with two dim lines, both of which are likely to show disappearances. A possible source of the effect found in Experiment 1 and replicated in Experiment 2 could therefore have been the increased difficulty of reporting two sets of disappearances rather than one. It could be hypothesized that although a subject may perceive equal disappearance of the reference line in each case, his accuracy of reporting will be greater when no other line disappearances have to be concurrently indicated, and that this was responsible for the apparent absence of interaction between bright and dim lines. Experiment 3 tested this hypothesis by means of the presentation of a series of simulated subjective disappearances of the bright line, thus giving it the phenomenal and response characteristics of a dim line. In addition, this procedure was expected to shed light on the probable accuracy of subjects' reports of naturally occurring subjective disappearances. Two stimulus configurations were used, equivalent to Conditions $A$ and $\mathrm{C}$ of Experiment 1 (see Figure 1). However, in this experiment, the bright line was presented with and without simulations, which were either to be reported or simply observed. This gave the set of five test conditions summarized in Table 3.

\section{Method}

Apparatus. The apparatus employed in Experiments 1 and 2 was modified so that the power supply to the lamps illuminating each stimulus channel could be interrupted according to a preset schedule. The latencies of subjects' responses to the start and end of simulations were recorded independently from measures of the duration and frequency of subjective disappearances.

Subjects. Ten subjects took part in the experiment. All had normal or corrected vision and no history of strabismus. 
Table 3

Summary of Conditions in Experiment 3

\begin{tabular}{ll}
\hline Con & \multicolumn{1}{c}{ Stimulus } \\
\hline A Two dim lines. & Response \\
B Dim reference line; bright second line. & Disappearances of either line reported. \\
C Dim reference line; bright second line with simulated subjective disappearances. Disappearance of either line reported. \\
D Dim reference line; bright second line with simulated subjective disappearances. Only disappearances of reference line reported. \\
\hline
\end{tabular}

Note-Con $=$ condition .

Procedure. All subjects received four sessions of testing, during each of which all five test conditions were employed. The first two sessions were carried out within a $1-\mathrm{h}$ period, following which there was an interval of at least $24 \mathrm{~h}$ before the last two sessions. Four simulated subjective disappearances of the bright line were initiated during each trial with Conditions $C$ and $D$. These simulations lasted $4 \mathrm{sec}$ each and began 30,55, 70, and $95 \mathrm{sec}$ after the start of the trial. The overall duration and frequency of simulations therefore approximated those which would be expected with subjective disappearances of a dim line.

\section{Results and Discussion}

The results could be classified in terms of either replications or stimulus conditions. Table 4a shows the results for each replication averaged over the four test conditions, and Table $4 \mathrm{~b}$ shows the results for each test condition averaged over the four replications. From Table 4a, it can be seen that the reference line was invisible for around $14 \mathrm{sec}$ in each session, with a mean period of about $3.5 \mathrm{sec}$. This consistency of response was borne out by an analysis of variance, which showed no significant effect of replication on either disappearance time or mean period and no interaction between replication and test condition. Piggins (1968) also found a high degree of consistency in repeated reports of the fragmentation of a square afterimage, although individual differences were marked. The results in Table $4 \mathrm{~b}$ show that in the presence of a bright line, whether or not it was subject to simulated subjective disappearances, the reference line disappeared for nearly twice as long as when it was paired with a line of equal luminance. This applied both when the simulated subjective disappearances were reported and when they were not. With Dunnett's procedure (Kirk, 1968), the amount by which the mean in Condition $A$ had to be exceeded in order to reach significance $(\mathrm{df}=3,27$, $p<.05$ ) was 6.6 for disappearance time and 1.2 for mean period. This was reached for each condition with disappearance time but not for Condition $\mathrm{C}$ with mean period. Overall, the contrast-dependent interaction of two steadily fixated lines was again apparent, but the absence of any difference between Conditions $B$ and $C$ showed that the disappearances of the reference line were not reduced when changes in the bright line also had to be reported. When the simulations were merely observed and not reported, there was still no increased interaction between the two lines. A stabilizing interaction was not, therefore, introduced by giving the bright line the same general visibility characteristics as would have been found with an equivalent dim line. This conclusion is supported by the finding of Atkinson (1972) for afterimages and of Swanston (1979) for steady fixation that the subjective disappearances of a line are not altered when those of a second line also have to be reported.

This experiment also provided information about the accuracy of subjects' reports of disappearances. Qualitatively, subjects could not readily distinguish between the simulations and other disappearances. At the end of the experiment, none indicated awareness of the artificial origin of the disappearances of the bright line. All subjects responded to each disappearance of the bright line, giving 160 responses in all. The mean reaction time to the start of a simulation was $666 \mathrm{msec}$ and $689 \mathrm{msec}$ to its end. Variation across successive replications was insignificant. These data correspond closely to those obtained for two subjects by Creighton, Tees, and Creighton (1972), who measured reaction time to report the simulated subjective disappearance of a single line viewed under steady fixation and found values of approximately $650 \mathrm{msec}$.

Insofar as the simulation procedure used here was functionally equivalent to subjective disappearances, it would appear that not only is accuracy of report acceptably high, but also that a consistent criterion of response can be maintained over a period of time. Schuck (1973) reported a similar level of accuracy for reports of more complex patterns of simulated fragmentation, provided the number of different fragments remained small. Evidence relating to potential sources of variation in the criterion of response to subjective disappearances is of value in the interpretation of many experiments with steady stimulation,

Table 4

Response Measures for the Stability of the Reference Line in Experiment 3

\begin{tabular}{|c|c|c|c|c|}
\hline & $\begin{array}{l}\text { ications } \\
\text { lst }\end{array}$ & 2nd & $3 r d$ & 4 th \\
\hline Disappearance Times (S) & 17.4 & 14.3 & 13.9 & 14.4 \\
\hline Mean Period (s) & 3.8 & 3.4 & 3.0 & 3.7 \\
\hline \multicolumn{5}{|c|}{ Test Condition } \\
\hline Disappearance Times (S) & 9.0 & 17.0 & 16.4 & 17.7 \\
\hline Mean Period (s) & 2.5 & 4.4 & 3.3 & 3.8 \\
\hline
\end{tabular}


particularly those in which the "meaningfulness" of a pattern has been held to determine its visibility (e.g., McKinney, 1963; Pritchard et al., 1960; Swanston, 1979).

\section{GENERAL DISCUSSION}

The results presented here indicate that the interaction between like-oriented steadily fixated lines is dependent on the relative contrast of the two. Similar orientation-selective interaction has been demonstrated for the phenomenal suppression of afterimages (Atkinson, 1972; Wade, 1976) and in binocular rivalry (Whittle et al., 1968). The stabilization of one line by another in the same orientation described here agrees with that demonstrated by Atkinson (1972) but does not show the same dependency on separation. That such interactions may be dependent on pattern features other than simple linear orientation has been shown by Taves and Atkinson (1973), who found that the visibility of a circular afterimage was increased in the presence of one or two additional afterimages of the same shape. The correspondence of findings with afterimages and steady fixation makes unlikely, but does not necessarily preclude, the possibility that different underlying mechanisms may be involved. In particular, it can be argued that any effects observed with steady fixation are the result of an appropriate pattern of involuntary eye movements (Cornsweet, 1971). In the present case, the contrast-selective interaction would require that eye movements be increased when both lines were of the same luminance, but equal for observation of one line or of two with differing contrast. Such an account cannot be conclusively disproved without concurrent measurements of eye movements, but there would not seem to be any a priori grounds for expecting the above outcome. Rather, a peripheral stimulus of higher contrast might be expected to induce a stronger tendency toward loss of fixation and thus a decreased possibility of disappearance of the reference line.

In general, studies of interaction in contrast threshold between adjacent visual stimuli have indicated that this is limited to small retinal separations, especially at photopic luminances (Westheimer, 1967). For example, Fiorentini and Mazzantini (1966) found that the threshold for an incremental line was elevated by a dark line within $.03 \mathrm{deg}$ but was lowered if the separation was between .06 and $.16 \mathrm{deg}$. Beyond this, no threshold modification was observed. Since their adaptation conditions were comparable to those of the present experiments, it is improbable that simultaneous contrast played a part in the interaction between steadily fixated lines of differing luminance.
One consequence of the presentation of two lines of differing luminance might have been expected to have produced an effect opposite to that observed. Insofar as a dim line is intermittently visible, it might also be supposed to have an intermittently stabilizing effect on another line.

Unless the physiological site of interactions between the two stimuli is different from that of whatever neural mechanism underlies the phenomenon of subjective disappearance, a permanently visible bright line should have a greater effect than one that disappears. However, there is evidence from studies on the influence of binocular rivalry on visual aftereffects that the effects of a stimulus are determined by the time for which it is physically present, rather than for the time for which it is subjectively visible to the observer (Blake \& Overton, 1979). Lehmkuhle and Fox (1975) found that the magnitude of the motion aftereffect was not influenced by the rivalous suppression of the inducing pattern, and a similar finding for the tilt aftereffect was reported by Wade and Wenderoth (1978). Such findings suggest that the site for the aftereffects is cortical, but not as central as that for binocular rivalry. These studies also show that it is necessary to have an independent measure of the effects of a subjectively invisible stimulus in order to infer their relative centrality to the processes underlying disappearance. In the present case, the processes underlying line interaction cannot be logically separated from those of disappearance, since the one is inferred from the other. The suggestion is that a dim line is more stable when paired with another dim line because the configuration has been identified as a pattern possessing a common brightness level, and that this occurs prior to whatever process produces the subjective disappearance of the lines. Such an account clearly supposes a central site for disappearances in steady fixation, but this site cannot be further identified on the basis of present information.

\section{REFERENCES}

AtKinson, J. Visibility of an afterimage in the presence of a second afterimage. Perception \& Psychophysics, 1972, 12, 257 262.

Atxinson, J. Properties of human visual orientation detectors: A new approach using patterned afterimages. Journal of Experimental Psychology, 1973, 98, 55-63.

Bishop, P. O., Coombs, J. S., \& Henry, G. H. Interaction effects of visual contours on the discharge frequency of simple striate neurones. Journal of Physiology (London), 1971, 219, 659-687.

Blake, R., \& Overton, R. The site of binocular rivalry suppression. Perception, 1979, 8, 143-152.

Brown, D. R., Schmidt, M. J., Fulaham, D. D., \& Cosorove, M. P. Human receptive field characteristics: Probe analysis of stabilized images. Vision Research, 1973, 13, 231-244.

Carpenter, R. H. S., \& Blakemore, C. Interactions between orientations in human vision. Experimental Brain Research, $1973,18,287-303$. 
Cornsweet, T. N. Visual perception. London: Academic Press, 1971.

Creighton, T. D., Tees, R. C., \& Creighton, D. E. Occipital EEG activity during fluctuations of perception under stabilized image and simplified stimulus conditions. Canadian Journal of Psychology, 1972, 26, 127-139.

Fionentini, A., \& Mazzantinı, L. Neural inhibition in the human fovea: A study of interactions between two line stimuli. Atti della Fondazione Giorgio Ronchi, 1966, 21, 738-747.

Forde, J., \& MacK Innon, G. E. Binocular stimulation and the fragmentation of afterimages. Quarterly Journal of Experimental Psychology, 1975, 27, 565-577.

KIRK, R. E. Experimental design: Procedures for the behavioral sciences. Belmont, Calif: Brooks/Cole, 1968.

LeHмkuHLE, S. W., \& Fox, R. The effect of binocular rivalry suppression on the motion aftereffect. Vision Research, 1975, 15, 855-859.

Leinowitz, H., Mote, F. A., \& Thurlow, W. R. Simultaneous contrast as a function of separation between test and inducing fields. Journal of Experimental Psychology, 1953, 46, 453-456.

MacKinnon, G. E., Forde, J., \& Piggins, D. J. Stabilized images, steadily fixated figures, and prolonged afterimages. Canadian Journal of Psychology, 1969, 23, 184-195.

McKinney, J. P. Disappearance of luminous designs. Science, $1963,140,403-404$.

Owen, D. H., \& Chalfant, C. Central integration effects with stabilized afterimages. Perception \& Psychophysics, 1970, 7, 284-286.

Pigains, D. J. Perception of a square viewed as a prolonged afterimage. Atti della Fondazione Giorgio Ronchi, 1968, 23, 149-172.

Pritchand, R. M., Heron, W., \& Hebb, D. O. Visual perception approached by the method of stabilized images. Canadian Journal of Psychology, 1960, 14, 67-77.

RATLIF, F. Mach bands: Quantitative studies on neural networks in the retina. San Francisco: Holden-Day, 1965.

Rosssel, F. P. Disappearances of semi-stabilized retinal images as a function of cell assemblies, field effects, and angle of observation (Doctoral dissertation, University of Minnesota, 1965). Dissertation Abstracts, 1966, 27, 1312-1313.

Schmid, M. J., Cosgrove, M. P., \& Brown, D. R. Stabilized images: Functional relationships among populations of orientation-specific mechanisms in the human visual system. Perception \& Psychophysics, 1972, 11, 389-392.

SchuCx, J. R. Factors affecting reports of fragmenting visual images. Perception \& Psychophysics, 1973, 13, 382-390.

Swanston, M. T. The effects of verbal meaning and response categorization on the fragmentation of steadily fixated patterns. Perception, 1979, 8, 635-646.

TAves, P., \& ATkinson, J. Visibility of an afterimage alone and in the presence of one or two additional afterimages. Perception \& Psychophysics, 1973, 13, 9-12.

Wade, N. J. Contour synchrony in binocular rivalry. Perception \& Psychophysics, 1973, 13, 423-425.

WADE, N. J. Monocular and dichoptic interaction between afterimages. Perception \& Psychophysics, 1976, 19, 149-154.

WADE, N. J. Why do patterned afterimages fluctuate in visibility? Psychological Bulletin, 1978, 85, 338-352.

WADE, N. J., \& WENDEROTH, P. The influence of color and contour rivalry on the magnitude of the tilt aftereffect. Vision Research, 1978, 18, 827-835.

Walker, P. Binocular rivalry: Central or peripheral selective processes? Psychological Bulletin, 1978, 85, 376-389.

Weisgtein, N. What the frog's eye tells the human brain: Single cell analyzers in the human visual system. Psychological Bulletin, 1969, 72, 157-176.

WEstheimer, G. Spatial interaction in human cone vision. Journal of Physiology (London), 1967, 190, 139-154.

Whittle, P., Bloor, D. C., \& Pocock, S. Some experiments on figural effects in binocular rivalry. Perception \& Psychophysics, 1968, 4, 183-188.

(Manuscript received January 2, 1980; revision accepted for publication June 10, 1981.) 